\title{
Síntomas de ansiedad y depresión en un grupo de pacientes oncológicos y en sus cuidadores
}

\author{
B. Rodríguez Vega, MD, Psiquiatra \\ A. Ortiz Villalobos, MD, Psiquiatra \\ A. Palao Tarrero, MD, Residente de Psiquiatría \\ C. Avedillo, MD, Residente de Psiquiatría \\ A. Sánchez-Cabezudo, MD, Residente de \\ Psiquiatría \\ C. Chinchilla, MD, Residente de Psiquiatría
}

Departamento de Psiquiatría, Hospital La Paz, Universidad Autónoma de Madrid, Madrid

ESPAÑA

RESUMEN - Objetivos: Determinar la prevalencia de los síntomas de ansiedad y depresión en un grupo de 534 pacientes oncológicos españoles y 371 cuidadores familiares.

Método: Se utilizó un cuestionario sociodemográfico así como la Escala de Ansiedad y Depresión Hospitalaria (HADS) con el fin de evaluar la presencia e intensidad de estos síntomas.

Resultados: Se observó que el 15,7\% de los pacientes dieron positivo en la subescala de ansiedad, incluida dentro de la escala de ansiedad y depresión hospitalaria (HADS),al utilizar un punto de corte mayor o igual a 11 y el 14,6\% lo hicieron en la subescala de depresión. En cuanto a los cuidadores familiares, el 36,4\% de ellos obtuvieron altas puntuaciones en la subescala de ansiedad y el 9,2\% lo hicieron en la subescala de depresión.

Conclusiones: Nuestros resultados confirman la alta prevalencia de los síntomas de ansiedad y depresión en ambos grupos y por lo tanto, la necesidad de diseñar intervenciones terapéuticas que incluyan de forma sistemática una evaluación familiar para contribuir a que los pacientes y sus familias puedan hacer frente a este tipo de problemas.

\section{Introducción}

Tras el estudio multicéntrico clásico de Derogatis (Derogatis et al. 1983) que demostraba que el $47 \%$ de los pacientes oncológicos presentan síntomas suficientes como para que se les pueda diagnosticar una enfermedad psiquiátrica, se han realizado numerosos estudios con el fin de detectar cuáles son estos síntomas (Razavi et al. 1990. Massie 1990, Payne et al. 1990,Berard 1998, McDaniel et al. 1995, Mermelstein 1992, Chochi- 
nov et al. 1994, Minagawa et al. 1996, Roth et al. 1998, Leopold 1998, Thompson 1998) y diseñar intervenciones terapéuticas específicas (Fawzy et al. 1990, Fawzy et al. 1990, Fawzy et al. 1993, Fawzy 1998, Greeer et al. 1992,Spiegel 1981).

Los trastornos mentales descritos más frecuentemente son las depresiones reactivas, los trastornos de ansiedad o ambos. Estas enfermedades son una constante entre las respuestas al estrés agudo, la ansiedad y la depresión. En el estudio de Derogatis (Derogatis et al. 1983), el $13 \%$ de los pacientes padecía trastornos afectivos, el $4 \%$ trastornos de ansiedad y un $68 \%$ trastornos de adaptación (con estado de ánimo deprimido, ansioso o con rasgos emocionales mezclados).

La importancia del descubrimiento de un enfoque terapéutico está basada en el hecho de que la mayoría de estas enfermedades reaccionan bien al tratamiento consiguiendo una mejora en la adaptación del paciente a los cambios, la calidad de vida, la sintomatología, e incluso, un posible efecto positivo en el curso de la enfermedad (Spiegel 1996, Endicott 1984). El psiquiatra, en la consulta de psico-oncología, tiene que enfrentarse a la tendencia común del paciente, la familia y el equipo médico a considerar los síntomas de ansiedad y depresión como parte de la respuesta esperada en una situación de enfermedad grave, y, por esta razón, no tienen en cuenta que estas reacciones puedan responder a un tratamiento adecuado (Endicott 1984).

El otro grupo de riesgo, que ha sido ampliamente mencionado en la literatura, lo constituyen los miembros de la familia de los pacientes. La investigación sobre la sobrecarga emocional que afecta a la persona que se convierte en el cuidador del paciente se ha convertido en uno de los objetivos más importantes de muchos estudios durante los pasados años (Kurtz et al. 1994, Nijboer 1998, Raveis 1998). Por un lado, se ha reconocido una mayor prevalencia de la sintomatología psiquiátrica en este grupo. Por otro lado, el periodo de sobrecarga para el cuidador se ha extendido, a medida que el tiempo de supervivencia del paciente aumenta gracias a los avances médicos (Raveis 1998). También se ha evaluado la importancia que tiene la familia a la hora de proporcionar apoyo emocional y social, lo que afecta a la adaptación del paciente a la enfermedad, su desarrollo y también a la posibilidad de recibir tratamiento en un contexto afín (Kurtz et al. 1994, Raveis 1998). El uso de instrumentos simples de detección para reconocer síntomas de ansiedad y depresión, tanto en los pacientes como en sus familias, facilita la identificación de los grupos de riesgo, los cuales podrían beneficiarse de tratamientos psicológicos y psiquiátricos (Razavi et al. 1990).

El objetivo principal de este estudio es determinar la prevalencia de los síntomas de ansiedad y depresión en un grupo de pacientes oncológicos extrahospitalarios y en sus cuidadores familiares en el Hospital La Paz de Madrid. Hemos elegido a esta población para llevar a cabo el estudio ya que la mayor parte del tratamiento oncológico se realiza de forma ambulatoria. Describimos las características de subgrupos de pacientes con puntuaciones que sobrepasan el punto de corte en lo que se refiere a los síntomas de ansiedad o depresión. La detección ha sido un paso previo al diseño de intervenciones terapéuticas específicas, actualmente en práctica, que serán el objetivo de nuestros próximos estudios.

\section{Material y métodos}

Se solicitó a seiscientos cuarenta y un pacientes que asistían a consultas externas de una clínica oncológica y al hospital de día de La Paz durante Mayo y Junio de 1999 que 
participasen en un estudio descriptivo que se estaba llevando a cabo. De ellos, 534 (83,3\%) aceptaron y rellenaron los cuestionarios de forma apropiada. 107 rechazaron participar porque no querían o se sentían incapaces de completar la Escala de Ansiedad y Depresión Hospitalaria (HADS). Se recogieron datos sociodemográficos tales como la edad y el sexo y no se descubrió ninguna diferencia entre los participantes y los no participantes, en lo que a estas variables se refiere.

El grupo de pacientes $(\mathrm{n}=534)$, y el miembro de la familia que dijo ser el cuidador de cada paciente $(n=371)$, completaban la relación clínica y socio-demográfica diseñada para este estudio y para la Escala de Ansiedad y Depresión Hospitalaria (HADS) (Payne 1999).

\section{Instrumentos de clasificación}

La Escala de Depresión y Ansiedad Hospitalaria (HADS) es un método de autovaloración que fue desarrollada por Zigmond y Snaith (Zigmond 1983) con el fin de detectar estados de depresión y ansiedad en un ámbito extrahospitalario. Está compuesto por dos subescalas de 7 items cada una, que diferencian perfectamente la depresión y la ansiedad. Durante el diseño de la escala, se intentaron excluir síntomas de autoreferencia -tales como migrañas o mareos-, o aquellos que pudieran ser de naturaleza psiquiátrica o somática -como el insomnio o anergia- debido a su utilización por el sector médico. El paciente tiene que completar la escala y reflejar en ella cómo se ha sentido la semana anterior.

La Escala de Depresión y Ansiedad Hospitalaria (HADS) ha demostrado tener las características psicométricas adecuadas (Moorey et al. 1991) y se ha utilizado extensamente en otros estudios (Razavi et al. 1990,
Payne et al. 1990, Berard 1998, Carroll et al. 1993). Ha sido comparada con otros métodos de medida tales como el GHQ y los resultados indican que existían pocas diferencias entre ambos cuando el personal médico era el encargado de aplicarlos (Lewis 1990). En los últimos años, la Escala de Depresión y Ansiedad Hospitalaria (HADS) ha sido validada para la población oncológica española (López-Roig et al. 1998, López Roig et al.).

Hemos utilizado los mismos puntos de corte que Carroll, esto es, 8 y 11, ya que han demostrado ser eficaces a la hora de detectar la ansiedad y los trastornos depresivos.

\section{Métodos estadísticos}

El análisis de los datos se realizó con el paquete estadístico SPSS para Windows 9.0. Los datos cuantitativos están descritos por medio de \pm DT $95 \%$ y se calcularon los intervalos de confianza de la prevalencia de ansiedad y depresión para las puntuaciones en la Escala de Depresión y Ansiedad Hospitalaria (HADS). La comparación entre los datos cuantitativos se llevó a cabo a través del test "chi-cuadrado". La correlación entre los datos cuantitativos se estudió con el coeficiente de correlación de Spearman. Se compararon los datos cuantitativos por medio del test de "TStudent" y el test de Mann-Whitney para datos que no son comunes.

\section{Resultados}

La tabla I muestra datos clínicos y sociodemográficos de un grupo de pacientes y cuidadores.

La tabla II muestra datos oncológicos de pacientes. 
Tabla I

Características de la muestra de pacientes y cuidadores

$\mathrm{N}=634$, Edad: Media 59.39/ 14.61(SD)

$\mathrm{N}$

\begin{tabular}{lrr}
\hline Hombres & 329 & $(51,9 \%)$ \\
Mujeres & 305 & $(48,1 \%)$ \\
Estado civil & 391 & $(68,7 \%)$ \\
$\quad$ Casados & 81 & $(14,2 \%)$ \\
$\quad$ Solteros & 75 & $(13,2 \%)$ \\
$\quad$ Viudos & 418 & $(82,4 \%)$ \\
Convivencia con pareja más de 20 años & & \\
Profesión & 143 & $(25,4 \%)$ \\
$\quad$ Ama de casa & 140 & $(24,9 \%)$ \\
$\quad$ Trabajador no cualificado & & \\
Profesión cónyuge & 176 & $(35,8 \%)$ \\
$\quad$ Ama de casa & 106 & $(22,2 \%)$ \\
$\quad$ Trabajador no cualificado & & \\
Tipo de convivencia & 135 & $(24,1 \%)$ \\
$\quad$ Pareja & 240 & $(33,6 \%)$ \\
Pareja e hijos & 467 & $(82,8 \%)$ \\
Sin antecedentes psiquiátricos & 106 & $(23,1 \%)$ \\
Creen poder beneficiarse de ayuda psquiátrica & 35 & $(6,4 \%)$ \\
Deseo de muerte & 22 & $(4 \%)$ \\
Ideas de suicidio & & \\
Cuidador & 350 & $(63,5 \%)$ \\
$\quad$ Cónyuge & 57 & $(10,3 \%)$ \\
Derivación a Psiquiatría & 42 & $(15,3 \%)$ \\
Rechazaron la derivación & & \\
Cuidador: & 350 & $(63,5 \%)$ \\
$\quad$ Pareja & 86 & $(15,6 \%)$ \\
Hijo & 27 & $(4,9 \%)$ \\
$\quad$ Madre & 276 & $(52,3 \%)$ \\
Pasa todo el día con el paciente & & \\
\hline &
\end{tabular}

Tabla II

Características oncológicas de la muestra de pacientes

\begin{tabular}{lrr}
\hline Tipo de tumor & & \\
Colon & 155 & $(24,3 \%)$ \\
Mama & 107 & $(16,8 \%)$ \\
Pulmón & 80 & $(12,5 \%)$ \\
Melanoma & 58 & $(9,1 \%)$ \\
Otorrinolaríngeo & 47 & $(7,4 \%)$ \\
Hodgkin & 31 & $(4,9 \%)$ \\
Urológico & 43 & $(6,7 \%)$ \\
Digestivo & 42 & $(6,6 \%)$ \\
Cabeza y cuello & 16 & $(2,5 \%)$ \\
Ginecológico & 3 & $(0,5 \%)$ \\
Otros & 56 & $(8,7 \%)$ \\
Metástasis & 238 & $(38,8 \%)$ \\
En activo & 408 & $(65,3 \%)$ \\
Tratamiento QT actual & 281 & $(50,6 \%)$ \\
En tratamiento radioterápico actual & 181 & $(48,9 \%)$ \\
Sin ingresos hospitalarios previos & 94 & $(25,7 \%)$ \\
Antecedentes oncológicos familiares & 227 & $(40,4 \%)$ \\
Entre 1 y 3 años desde el diagnóstico & 457 & $(73 \%)$ \\
\hline
\end{tabular}


Las puntuaciones sobre la Escala de Depresión y Ansiedad Hospitalaria para pacientes y cuidadores aparecen en la tabla III. En cuanto a los dos puntos de corte de la ansiedad, los cuidadores obtienen puntuaciones mayores que el colectivo de pacientes. No ocurre lo mismo con la depresión, especialmente con los síntomas más agudos. Al comparar al grupo de pacientes que tienen puntuaciones superiores a 8 y 11 con el grupo de pacientes con puntuaciones inferiores a los puntos de corte, se demostró que tenían una edad homogénea, excepto en la subescala de depresión, donde las diferencias eran estadísticamente significativas (p.....0,005). Aquellos que tenían una puntuación inferior a 8 para la depresión $(\mathrm{n}=$ 394), tenían una edad media de 57,82 años, los que habían obtenido una puntuación superior a $8(\mathrm{n}=140)$ tenían una edad media de 62,2. Quienes obtuvieron una puntuación inferior a 11, tenían una edad media de 58,27, mientras que aquellos con una puntuación superior a 11 en la misma subescala de depresión tenían una edad media de 63,06. Resumiendo, los pacientes con mayor número e intensidad de síntomas depresivos eran más mayores.

\section{Grupo de pacientes}

La tabla IV muestra las características más importantes de los pacientes recogiendo puntuaciones superiores a los puntos de corte en cualquiera de las subescalas incluidas en la de la Escala de Ansiedad y Depresión Hospitalaria (HADS). Las puntuaciones superiores eran más frecuentes en mujeres, exceptuando la depresión donde el punto de corte es más bajo, sin obtener resultados estadísticos significativos. Los pacientes casados, que convivían con su cónyuge e hijos, fueron los que consiguieron mayor puntuación en la subescala de ansiedad pero no en la subescala de depresión. Los síntomas depresivos que son superiores a los puntos de corte eran más frecuentes en pacientes jubilados.

No surgieron datos significativos sobre la localización del tumor o la presencia de enfermedades concomitantes. Sin embargo, la presencia de metástasis diferenciaba al subgrupo en donde la sintomatología depresiva era más frecuente. Los pacientes que han terminado recientemente la quimioterapia (menos de un mes) obtuvieron puntua-

Tabla III

Valores del HADS en pacientes y cuidadores

\begin{tabular}{lrrr} 
Pacientes & N 534 & $\%$ & IC 95\% \\
\hline HADSA8 & 176 & $33 \%$ & $29-37$ \\
HADSA11 & 84 & $15,7 \%$ & $12,8-19$ \\
HADSD8 & 140 & $26,2 \%$ & $22,6-30,1$ \\
HADSD11 & 78 & $14,6 \%$ & $11,7-17,8$ \\
Cuidadores & N 371 & & \\
HADSA8F & 209 & $56,3 \%$ & $51,1-61,3$ \\
HADSA11F & 135 & $36,4 \%$ & $31,5-41,4$ \\
HADSD8F & 86 & $23,2 \%$ & $19-27,7$ \\
HADSD11F & 34 & $9,2 \%$ & $6,5-12,4$ \\
\hline
\end{tabular}

HADSA = HADS ansiedad.

$\mathrm{F}=$ Cuidador familiar.

HADSD $=$ HADS depresión. 
Tabla IV

Características de los pacientes y valores del HADS

\begin{tabular}{lcccc} 
& HADSA8 & HADSA11 & HADSD8 & HADSD11 \\
\hline $\mathrm{N}=534$ & $176(33 \%)$ & $84(15,7 \%)$ & $140(26,2 \%)$ & $78(14,6 \%)$ \\
\hline Sexo & & & & \\
$\quad$ Hombres & $71(25,6 \%)$ & $31(11,2 \%)$ & $65(23,4 \%)$ & $32(11,5 \%)$ \\
$\quad$ Mujeres & $105(40,9 \%)^{*}$ & $53(20,6 \%)^{*}$ & $75(29,3 \%) \mathrm{ns}$ & $46(18 \%)^{*}$ \\
Estado civil & & & \\
$\quad$ Casado o conviviendo & $133(35,8 \%)^{*}$ & $67(18 \%)^{*}$ & $97(26 \%) \mathrm{ns}$ & $50(13,4 \%) \mathrm{ns}$ \\
$\quad$ Soltero, viudo o divorciado & $43(26,5 \%)$ & $17(10,5 \%)$ & $43(26,9 \%)$ & $28(17,5 \%)$ \\
Situación laboral del paciente & & & & \\
$\quad$ Jubilados & $47(28 \%) \mathrm{ns}$ & $26(15,5 \%) \mathrm{ns}$ & $58(34,3 \%)^{*}$ & $20,1 \% *$ \\
Tipo de convivencia: & & & & \\
$\quad$ Con padres, pareja e hijos & $6(100 \%)^{*}$ & $5(83,3 \%)^{*}$ & $2(33,3 \%) \mathrm{ns}$ & $1(16,7 \%) \mathrm{ns}$ \\
$\quad$ Con otros familiares & $170(32,3 \%)$ & $79(15 \%)$ & $137(26 \%)$ & $77(14,6 \%)$ \\
Existencia de metástasis & $73(35,3 \%) \mathrm{ns}$ & $35(16,9 \%) \mathrm{ns}$ & $77(36,8 \%)^{*}$ & $44(21,1 \%)^{*}$ \\
Terminar el tto QT & & & & \\
$\quad$ Hace menos de un mes & $9(64,3 \%)^{*} 5(35,7 \%) \mathrm{ns} \mathrm{p}=0,638(57,1 \%)^{*}$ & $5(35,7 \%)^{*}$ \\
$\quad$ Más de un mes & $150(33,5 \%)$ & $71(15,8 \%)$ & $111(24,8 \%)$ & $60(13,4 \%)$ \\
Sin pensamientos de muerte o suicidio & $89(19,9 \%)^{*}$ & $59(13,2 \%)^{*}$ & $89(19,9 \%)^{*}$ & $41(9,2 \%)^{*}$ \\
Deseo de muerte & $35(58,3 \%)^{*}$ & $15(25 \%)^{*}$ & $35(58,3 \%)^{*}$ & $24(40 \%)^{*}$ \\
Ideas de suicidio & $14(66,7 \%)^{*}$ & $10(47,6 \%)^{*}$ & $14(66,7 \%)^{*}$ & $13(61,9 \%)^{*}$ \\
Antecedentes psiquiátricos de depresión & $24(61,5 \%)^{*}$ & $16(41 \%)^{*}$ & $19(48,7 \%)^{*}$ & $14(35,9 \%)^{*}$ \\
Creen que se beneficiarían de ayuda psiquiátrica $62(60,8 \%)^{*}$ & $34(33 \%)^{*}$ & $62(60,8 \%)^{*}$ & $30(29,4 \%)^{*}$ \\
Derivación adecuada a Psiquiatría & $28(52,8 \%)^{*}$ & $18(34 \%)^{*}$ & $23(43,4 \%)^{*}$ & $19(35,8)^{*}$ \\
Derivan a Psiquiatría con & & & & \\
$\quad$ HADS menor del punto de corte & $25(47,2 \%) \mathrm{ns}$ & $35(66 \%)^{*}$ & $30(56,6 \%) \mathrm{ns}$ & $34(64,2)^{*}$ \\
No se deriva con & & & & \\
$\quad$ HADS mayor del punto de corte & $147(30,9 \%)^{*}$ & $65(13,7 \%)^{*}$ & $116(24,4 \%)$ & $58(12,2 \%)^{*}$ \\
No se deriva con & $329(69,1 \%)$ & $411(86,3 \%)$ & $359(75,6 \%)$ & $417(87,8 \%)$ \\
$\quad$ HADS menor del punto de corte & & & & \\
\hline
\end{tabular}

(*) $\mathrm{p}<0,05$.

ciones más altas en casi todas las escalas. Algunos pacientes afirmaron no haber pensado en la muerte o tenido ideas suicidas en ambas subescalas. Con bastante frecuencia, los pacientes se referían a ideas sobre la muerte o a ideas suicidas elaboradas incluso cuando tenían puntuaciones bajas en depresión o ansiedad (entre 8 y 10). Las personas con historia psiquiátrica, elaborada por un profesional, mostraron un mayor número y una mayor intensidad de síntomas depresivos y de ansiedad, con una probabilidad estadística significativa. Estos pacientes también creían que podían beneficiarse de ayuda psiquiátrica y psicológica, con una frecuencia significativamente mayor a aque- llos que estaban por debajo de cualquiera de los puntos de corte.

Siempre que el cuidador era un hijo o una hija en lugar del cónyuge, de un familiar o de una persona allegada, las puntuaciones eran igual o superiores a 11 tanto en la subescala de ansiedad ( $\mathrm{n}=19,24,7 \%)$ como en la subescala de depresión $(\mathrm{n}=17$, $22,4 \%$ ). Pasar todo el día con el paciente también fue asociado con una mayor frecuencia de sintomatología depresiva, con puntuaciones superiores a $8(\mathrm{n}=84,32,4 \%)$ o superiores a $11(\mathrm{n}=47,18,1 \%)$.

Entre el $35 \%$ y casi el $53 \%$ de los pacientes a los que el oncólogo recomendó aten- 
ción psiquiátrica, fueron remitidos correctamente. Esto es, se envió a los pacientes cuando realmente mostraban síntomas de ansiedad o depresión. Estos datos no tienen en cuenta las veces que los pacientes fueron remitidos a causa de una sintomatología diferente a la ansiedad o la depresión, tales como sucede en el caso del alcoholismo. Un dato aún más significativo es el que se refiere a los pacientes que no han sido derivados a Psiquiatría y tienen puntuaciones superiores a los puntos de corte en la Escala de Ansiedad y Depresión Hospitalaria, oscilando entre el $12 \%$ para una sintomatología más grave y el $30 \%$ para síntomas leves.

\section{Grupo de cuidadores}

En el grupo de cuidadores con síntomas de ansiedad y depresión (tabla V), la presencia de mujeres era significativamente más importante en todas las escalas, excepto en aquélla que correspondía a los síntomas depresivos más intensos (por encima del punto de corte 11).

No se hallaron diferencias significativas en variables tales como el estado civil, la profesión o la situación laboral, ni tampoco en las variables que se refieren a la localización del tumor o a la presencia de enfermedades concomitantes. Los cuidadores presentaban sintomatología depresiva con más frecuencia si el cáncer del paciente era metastásico, algo que también ocurría en el caso de los pacientes.

La correlación existente entre la subescala de ansiedad y la de depresión, en el grupo de pacientes y en el de cuidadores, así como también la posible correlación que se ha realizado de cada subescala para ambos grupos que se presenta en la tabla VI muestra resultados significativos. La subescala de ansiedad está correlacionada con la subescala de depresión tanto en el grupo de pacientes como en el de las de familias. También se observó que existía correlación entre las subescalas de ansiedad y depresión en pacientes y las subescalas de ansiedad y depresión en familias.

Tabla V

Características de los cuidadores y valores del HADS

\begin{tabular}{lcccc} 
& HADSA8 & HADSA11 & HADSD8 & HADSD11 \\
\hline $\mathrm{N}=371$ & $209(56,3 \%)$ & $135(36,4 \%)$ & $86(23,2 \%)$ & $34(9,2 \%)$ \\
\hline Sexo & & & & \\
$\quad$ Mujeres & $145(68,1 \%)^{*}$ & $101(47,4 \%)^{*}$ & $67(31,5 \%)^{*}$ & $24(11,3 \%) \mathrm{ns}$ \\
$\quad$ Hombress & $64(40,5 \%)$ & $34(21,5 \%)$ & $19(12 \%)$ & $10(6,3 \%)(\mathrm{p}=0,14)$ \\
Existencia de metástasis & $99(60 \%) \mathrm{ns}$ & $68(41,2 \%) \mathrm{ns}$ & $47(28,5 \%)^{*}$ & $23(13,9 \%)^{*}$ \\
\hline
\end{tabular}

(*) $\mathrm{p}<0,05$.

Tabla VI

Coeficientes de correlación (Rho de Spearman)

\begin{tabular}{lcccc} 
& HADSA & HADSD & HADS AF & HADS DF \\
\hline HADSA & 1 & & & \\
HADSD & $445^{*}$ & 1 & & \\
HADS AF & $241^{*}$ & $162^{*}$ & 1 & \\
HADS DF & $156^{*}$ & $360^{*}$ & $596^{*}$ & \\
\hline
\end{tabular}

(*) La correlación es significativa al nivel 0,01 bilateral. 


\section{Discusión/Conclusión}

Que nosotros sepamos,éste es el primer y más extenso estudio español sobre síntomas emocionales en pacientes oncológicos después de que se haya validado la versión española de la Escala de Ansiedad y Depresión Hospitalaria (HADS) [López-Roig et al. 1998, López-Roig (15)].

Al igual que en el estudio de Carroll (Caroll et al. 1993), no se realizaron entrevistas psiquiátricas posteriores a pacientes que habían obtenido puntuaciones altas en cualquiera de las dos Escalas de Depresión y Ansiedad Hospitalaria (HADS). Por lo tanto, no podemos determinar cuántos pacientes pudieron beneficiarse de una intervención psiquiátrica.

Al utilizar un punto de corte inferior (mayor o igual a 8) en la muestra de pacientes, descubrimos que un $33 \%$ de los pacientes $(n=176)$ daban positivo en la escala de ansiedad y un $26,2 \%(n=140)$ en la escala de depresión. Al utilizar el punto de corte superior (mayor o igual a 11), vimos que un $15,7 \%(\mathrm{n}=84)$ de los pacientes lo daban en la escala de ansiedad y un $14,6 \%(n=78)$ en la escala de depresión. Por lo tanto, al utilizar el punto de corte inferior, se justificaría la realización de un examen psiquiátrico más profundo al 56,2\% de esta población. Esta cifra es más alta que la descubierta por Carroll (Carroll et al. 1993) (47\%) o por Derogatis en su estudio multicéntrico (Derogatis 1983) (47\%), a pesar de que nuestro grupo está compuesto únicamente por pacientes procedentes de consultas externas, cuyos síntomas de intensidad del dolor tienden a ser inferiores a los de los pacientes que permanecen ingresados (Mermelstein 1992). Al utilizar el punto de corte superior, descubrimos que sería necesario que el $30,3 \%$ de los pacientes se sometiera a este examen.

En un estudio anterior (Leopold et al. 1998), utilizando otro instrumento de evaluación (PRIME-MD), se descubrió una prevalencia de ansiedad y depresión de casi el $50 \%$ en un grupo de pacientes que recibían un tratamiento radioterápico. Ha de tenerse en cuenta que en nuestro grupo, el $50 \%$ de los pacientes recibían quimioterapia o radioterapia. Puede que éste sea uno de los factores que han contribuido a la alta prevalencia descubierta.

Un gran número de mujeres que dieron positivo en todas las subescalas pudieron reflejar una mayor frecuencia de los síntomas de ansiedad y depresión de lo que ellas mostraban o decían tener.

Como sucede en otros estudios (Massie 1990, McDaniel et al. 1995, Chochinov et al. 1994, Minagawa et al. 1996), la sintomatología depresiva era más frecuente cuando estaba relacionada con una edad media más avanzada y se daba una presencia de metástasis. En otros estudios, la mayor prevalencia de depresión se presenta en pacientes en estadíos terminales (Massie 1990) y se ha señalado también la gravedad de la enfermedad como un factor de riesgo de depresión (McDaniel et al. 1995, Chochinov et al. 1994, Minagawa et al. 1996). Sin embargo, los resultados que están relacionados con los efectos de la edad y la etapa evolutiva en la que se encuentra la enfermedad son contradictorios. En este sentido, el estudio de Schnoll (Schnoll et al. 1998) es instructivo, ya que integra descubrimientos contradictorios ilustrando el papel mediador de un coping style con un grupo de mujeres a las que se les diagnosticó cáncer de mama.

En estudios anteriores (Massie 1990, McDaniel et al. 1995, Mermelstein 1992) se había mencionado la presencia frecuente de 
trastornos de adaptación una vez que los pacientes terminaban el severo tratamiento al que se habían sometido. En nuestro estudio,la mayor presencia de ansiedad y depresión un mes después de terminar el tratamiento quimioterápico, podía reflejar los descubrimientos anteriores. Cuando ha pasado un tiempo tras la finalización del tratamiento, esta asociación deja de existir y tampoco es posible encontrarla cuando el paciente está recibiendo tratamiento. La explicación de los propios pacientes, cuando, en la consulta, informaban sobre sus sentimientos de "falta de protección" o "desamparo" tras terminar un tratamiento muy agresivo que, al mismo tiempo "llevaba a cabo un ataque frontal contra su enfermedad", podría explicar los hallazgos mencionados anteriormente.

El cáncer es una enfermedad muy importante que influye en muchos aspectos no sólo al paciente sino también a sus familiares más cercanos. Algunos estudios han mostrado que los pacientes y sus acompañantes presentaban niveles más altos de psicopatología si se les comparaba con la población general, y no existían diferencias significativas entre los pacientes y sus acompañantes en lo que se refería a los niveles de angustia (Nijboer et al. 1998, Raveis 1998).

También descubrimos una alta prevalencia de ansiedad y síntomas depresivos en los grupos de cuidadores familiares. Al utilizar un punto de corte inferior (mayor o igual a 8), descubrimos que un 56,3\% $(n=209)$ de los pacientes daban positivo en la escala de ansiedad y un $23,2 \%(n=86)$ en la escala de depresión. Al utilizar el punto de corte superior (mayor o igual a 11), descubrimos que el $36,4 \%(n=135)$ de los pacientes que habían dado positivo en la escala de ansiedad y el 9,2\% ( $n=34)$ lo habían hecho en la escala de depresión.
En cuanto a los síntomas presentes en los grupos de cuidadores, consideramos que nuestros datos son muy importantes ya que utilizamos la Escala de Depresión y Ansiedad Hospitalaria para cuantificarlos. Suponiendo que hubiéramos utilizado otra escala que no excluyera los síntomas somáticos, imaginamos que habríamos descubierto una mayor prevalencia en el grupo de cuidadores familiares.

Al igual que en el grupo de pacientes, la presencia de metástasis está íntimamente relacionada con los síntomas de depresión en los cuidadores. Otros estudios han obtenido resultados similares (Kurtz et al. 1994). Al tiempo que aumenta la gravedad de la enfermedad, los cuidadores experimentaban niveles más altos de depresión, reflejando la situación de los pacientes. Este dato refuerza la impresión clínica de que una enfermedad que amenaza la vida representa un desafío para la estabilidad emocional del paciente de su familia y afecta al ambiente social que le rodea. Por lo tanto, nuestros resultados confirman la necesidad de considerar a los cuidadores de pacientes oncológicos como una población de alto riesgo en cuanto a la morbilidad psiquiátrica se refiere $y$, a consecuencia de ello, surge la necesidad de incluirles en los protocolos de recomendación, teniéndoles en cuenta e incluyéndoles en el tratamiento.

Es posible que la alta prevalencia de los síntomas de ansiedad y depresión en los grupos de pacientes y familiares podría incluir un porcentaje falso positivo relacionado con reacciones agudas de estrés que pueden dar lugar a puntuaciones altas en la Escala de Depresión y Ansiedad Hospitalaria. Además, como comentó Razavi (Razavi et al. 1990), la angustia expresada no siempre tiene que estar correlacionada con la presencia de un determinado trastorno. Un aspecto común a todos los pacientes con enfermeda- 
des crónicas es que presentan síntomas angustiosos e inoportunos pero no reúnen criterios suficientes como para que se pueda decir que padecen una enfermedad mental. Estos síntomas no específicos son un área crítica en el estudio de pacientes (Roth et al. 1998). Razavi (Razavi et al. 1990) utilizó puntos de corte superiores y descubrió que cuando el punto de corte era de 13, la prueba tenía un $75 \%$ de sensibilidad y un $75 \%$ de especificidad. Payne (Payne et al. 1999) descubrió que, al utilizar un punto de corte de 10 o más en un grupo de mujeres enfermas de cáncer de mama que acudían a consultas externas, se debía realizar un examen psiquiátrico más profundo al $33 \%$ de ellas. Berard (1998), utilizando un punto de corte de 8 en la subescala de depresión, descubrió una prevalencia del $14 \%$ en lo que a trastornos depresivos se refiere aunque el autor admite la posibilidad de que no se haya dado la importancia suficiente a esta cifra debido a dificultades de aleatoriedad en este estudio. López Roig et al. (1998), en un grupo de pacientes oncológicos españoles, descubrió una sensibilidad y una especificidad de alrededor de 0,80 utilizando puntos de corte de 7 para la ansiedad y de 4 para la depresión. Mermelstein y Lesko (1992) concluyeron que la mayoría de los estudios citan los porcentajes de prevalencia para síntomas depresivos del 20-25\%, que están más próximos a nuestros resultados de $26,2 \%$ al utilizar un punto de corte de 8 .

En el grupo de pacientes que hemos estudiado, son frecuentes los deseos de morir o suicidarse, incluso en los puntos de corte más bajos de ambas escalas. Posiblemente, este resultado refleja la dificultad de aceptar este tipo de pensamientos en nuestro medio, cuando la persona presenta una sintomatología grave (por ejemplo, con puntos de corte superiores a 11) y tiene mayor capacidad para aceptar ideas sobre la muerte, tan fre- cuentes tanto en pacientes como en cuidadores, sin que por ello muestre síntomas graves de ansiedad o depresión (como ocurre cuando en ambas subescalas, los puntos de corte están entre 8 y 10).

La mayoría de los pacientes que creían poder beneficiarse de tratamiento psicológico o psiquiátrico, obtuvieron las puntuaciones más bajas en ambas subescalas. Los pacientes con puntuaciones superiores eran aquellos que pensaban que el tratamiento psiquiátrico no podría ayudarles. Se podría asumir por tanto, que sentir la necesidad de someterse a un tratamiento psiquiátrico no indica de forma adecuada la intensidad de los síntomas de ansiedad y depresión del paciente.

Por otro lado, de todos los pacientes que el oncólogo deriva a una consulta psiquiátrica, gran parte de ellos tienen puntuaciones inferiores al punto de corte en la Escala de Ansiedad y Depresión Hospitalaria. Como se ha señalado anteriormente, este hecho podría ser atribuido a varios factores. En primer lugar, podría reflejar una proporción de pacientes que han sido orientados de forma adecuada, pero que no han obtenido puntuaciones altas en lo que a los síntomas de ansiedad y depresión se refiere. Como hemos visto, otra explicación posible es que la percepción subjetiva de la necesidad de una consulta no estaba relacionada con la intensidad de la sintomatología, y podía ser el propio paciente quien sugiriera al oncólogo directa o indirectamente, a dónde debía ser derivado (Passik et al. 1998) sin que estos síntomas existiesen. Otra posible explicación se basa en la prioridad que establece el oncólogo a la hora de asegurar una orientación adecuada a los pacientes con una patología más grave en lo que a la ansiedad o depresión se refiere, antes que su derivación por problemas leves. 
Ante todo, los médicos están preocupados por el bienestar de sus pacientes y prefieren realizar más diagnósticos de los que deberían a pasar por alto una enfermedad grave. Este último punto puede basarse en el hecho de que los oncólogos no sólo remiten un pequeño porcentaje de pacientes que presenta una patología grave en lo que a los síntomas de ansiedad y depresión se refiere, ya que también derivan a muchos pacientes cuyas puntuaciones son inferiores a los puntos de corte. Este hecho coincidiría con el descubierto por Passik (Passik et al. 1998) quien afirma que los oncólogos están más influenciados por síntomas como el llanto o un estado de ánimo depresivo y datos médicos que son útiles pero que sin embargo no son los indicadores más fiables de depresión en este tipo de población. En conclusión, los oncólogos son más exactos en lo que respecta a los síntomas de ansiedad y depresión cuando deciden no remitir al paciente a una consulta psiquiátrica que cuando lo hacen.

La fuerte correlación descubierta entre las subescalas de ansiedad y depresión, tanto en el grupo de pacientes como en el de cuidadores nos hace dudar si estamos identificando a dos tipos de poblaciones diferentes o si se han creado dos etiquetas para un mismo grupo. En otros estudios también se descubrió una fuerte correlación entre estas dos subescalas (Carroll et al. 1993). La correlación descubierta entre las puntuaciones de los pacientes y los cuidadores también sostiene la idea clínica de que la enfermedad no es sólo un proceso individual sino que también es relacional y profundo. Cuando el paciente muestra síntomas de ansiedad o depresión, existe una alta probabilidad de que el cuidador, a menudo un familiar, también las padezca.

La Escala de Ansiedad y Depresión Hospitalaria es un instrumento muy útil para investigar la ansiedad y la depresión en pacientes que tienen cáncer, que ya había sido establecida con anterioridad en estudios de población española y anglo-sajona (Razavi et al. Payne et al. 1990, Berard 1998, Roth et al. 1998, Lewis 1990, LópezRoig et al. 1998, López-Roig et al. 1998) aunque no se puede utilizar para el diagnóstico. En nuestro estudio, la alta prevalencia de ansiedad que sufren tanto pacientes como cuidadores refuerza la necesidad de un examen psiquiátrico más profundo con el fin de llevar a cabo intervenciones terapéuticas en esta población.

\section{Bibliografía}

BERARD, R.M.F.,BOERMEESTER, F.,VILJOEN, G. Depressive disorders in an out patient oncology setting: Prevalence assessment and management. Psycho-oncolo gy, 7:112-120, 1998.

CARROLL, B.T.,KATHOL,R.G.,NOYES, R., WALD, T.G.,Clamon,gh. Screening for depression and anxiety in cancer patients using the Hospital Anxiety and Depression Scale. Gen Hosp Psychiatry, 15: 69-74, 1993.

CHOCHINOV, H.M., WILSON, K.G., ENNS, M., LANDER, S. Prevalence of depression in the terminally ill: Effects of diagnostic criteria and symptom threshold judgements. Am J Psychiatry, 151: 537-540, 1994

DEROGATIS, L.R., MORROW, G.R., FETTING, J. et $a l$. : The prevalence of psychiatric disorders among cancer patients. JAMA, 249: 751-757, 1983.

ENDICOTT, J. Measurement of depression in patients with cancer. Cancer, 53 (Supp): 2243-2249, 1984.

FAWZY, F.I., COUSINS, N., FAWZY, N.W., KEMENY, M.E., ELASHOFF, R., MORTON, D. A Structured Psychiatric Intervention for Cancer Patients. I. Changes Over Time in Methods of Coping and Affective Disturbance. Arch Gen Psychiatry, 47: 720-725, 1990.

FAWZY, F.I. FAWZY, N.W. Canada A. Psychosocial treatment of cancer:an update. Current Opinion in Psychi atry, 11: 601-605, 1998.

FAWZY, I.F., FAWZY, N.W.,HYUN, C.S.,ELASHOFF, R.,GUTRIE, D., FAHEY, J.L.,MORTON, D.L. Malignant Melanoma. Effects of an Early Structured Psychiatric 
Intervention, Coping, and Affective State on Recurrence and Survival 6 Years Later. Arch Gen Psychiatry, 50: 681$689,1993$.

FAWZY, F.I., KEMENY, M., FAWZY, N.W., ELASHOFF, R., MORTON, D., COUSINS, N., FAHEY, J.L. A Structured Psychiatric Intervention for Cancer Patients. II.Changes Over Time in Immunological Measures. Arch Gen Psychiatry, 47: 729-735, 1990.

GREER, S., MOOREY, S., BARUCH, J.D.R., WATSON, M., ROBERTSON, B.M.,MASON, A., ROWDEN, L., LAW, M.G., BLISS, J.M. Adjuvant psychological therapy for patients with cancer: a prospective randomised trial. BMJ, 304:675-679,1992.

KURTZ, M.E., GIVEN, B., KURTZ, J.C., GIVEN, C.W. The interaction of age, symptoms and survival status on physical and mental health of patients with cancer and their families. Cancer, 1: 74, n. ${ }^{\circ}$ 7: 2071-2077, 1994.

LEOPOLD, K.A., AHLES, T.A., WALCH, S. et al. Prevalence of mood disorders and utility of the PRIMEMD in patients undergoing radiation therapy. Int. J. Radia tion Oncology Biol Phys, 42, n. ${ }^{\circ}$ 5, 1105-1112, 1998.

LEWIS, G.,WESSELEY, S. Comparison of the General Health Questionnaire and the Hospital anxiety and depression scale. Br J Psychiatry, 157: 860-864, 1990.

LÓPEZ-ROIG, S., TEROL, M.C., NEIPP, M.C., LEYDA, I., MASSUTÍ, B., PICÓ, C., PASTOR, M.A. Assesing anxiety and depression with HADS scale in a spanish cancer population:A preliminary validation study. Psycho-oncology, 7, 1998.

LÓPEZ-ROIG, S., TEROL, M.C., PASTOR, M.A., RODRÍGUEZ-MARÍN, J., NEIPP, M.C., LEYDA, J.I. MARTÍN-ARAGÓN, M. Ansiedad y Depresión en pacientes oncológicos. Ansiedad y estres (in press).

MASSIE. M.J., HOLLAND. J,C. Depression and the cancer patient. J Clin Psychiatry, 75: 12-17, 1990.

McDANIEL, J.S., MUSSELMAN, D.L., PORTER, M.R., REED, D.A., NEMEROFF, D.B. Depression in patients with cancer: diagnosis, biology and treatment. Arch Gen Psychiatry, 52: 89-97, 1995.

MERMELSTEIN, H.T., LESKO, L. Depression in patients with cancer. Psycho-oncology, 1: 199-215, 1992.

MINAGAWA, H., UCHITOMI, Y., YAMAWAKI, S., ISHITANI, K. Psychiatric morbidity in terminally ill cancer patient. Cancer, 78: 5, 1131-1137, 1996.

MOOREY, S.,GREER, S., WATSON, M. et al. The factor structure and factor stability of the Hospital Anxiety and Depression Scale. Br J Psychiatry, 158:255-259,1991.
NIJBOER,C. et al. Cancer and caregiving: The impact on the caregiver's health. Psycho-oncology, 7: 3-13, 1998.

PASSIK, S.D., DUGAN, W., McDONALD, M.V., ROSENFELD, B., THEOBALD, D.E., EDGERTON, S. Oncologists' recognition of depression in their patients with cancer. J Clin Oncol, 16: 1594-160, 1998.

PAYNE, D.K., HOFFMAN, R.G., THEODOULOU, M.,DOSIK,M., MASSIE,M.J. Screening for anxiety and depression in women with breast cancer. Psychosom, 40:1, 64-69, 1999.

RAVEIS, V.H.,KARUS, D.G.,SIEGEL, K. Correlates of depressive symptomatology among adult daughter caregivers of a parent with cancer. Cancer. 83:8,1652-1663,1998.

RAZAVI, D., DELVAUX, N., FARVACQUES, C., ROBAYE, E. Screening for adjustment disorders and major depressive disorders in cancer inpatients. Br J Psy chiatry, 156: 79-83, 1990.

ROTH,A.J., KORNBLITH,A.B., BATEL-COPEL, L., PEABODY, E., SCHER, H.I., HOLLAND, J. Rapid screening for psychological distress in men with prostate carcinoma. Cancer, 82: n. ${ }^{\circ}$ 10, 1998.

SCHNOLL, R.A., HARLOW, L.L., STOLBACH, L., BRANDT, U. A structural model of the relationships among stage of disease, age, coping and psychological adjustment in women with breast cancer. Psycho-oncology, 7: 69-77, 1998.

SPIEGEL, D., BLOOM, J.R., YALOM, I. Group support for patients with metastatic cancer. Arch Gen Psychia try, 38: 527-533, 1981.

SPIEGEL, D. Cancer and depression. Br J Psychiatry, 168 (suppl 30): 109-116, 1996.

THOMPSON, D.S., SHEAR, K. Psychiatric disorders and gynecological oncology: a review of the literature. General Hospital Psychiatry, 20: 241-247, 1998.

ZIGMOND, A.S., SNAITH, R.P. The Hospital Anxiety and Depression Scale. Acta Psychiat. Scand, 76:361-370, 1983.

Dirección para correspondencia:

Dr. B. Rodríguez Vega, MD

Departamento de Psiquiatría

Hospital La Paz

Paseo de la Castellana, 261

28046 Madrid

Tel. 72772 76,

e-mail: brvega@terra.es

ESPAÑA 